\title{
EL JUEZ CONSTITUCIONAL DE SUDÁFRICA DECLARANDO LA INCONSTITUCIONALIDAD DE LA DENUNCIA POR EL PODER EJECUTIVO DEL TRATADO DE ROMA SOBRE LA CORTE PENAL INTERNACIONAL, AL HABER SIDO ADOPTADA SIN LA APROBACIÓN PREVIA DEL PARLAMENTO
}

\author{
ALLAN R. BREWER-CARÍAS \\ Universidad Central de Venezuela \\ allanbrewercarias@gmail.com
}

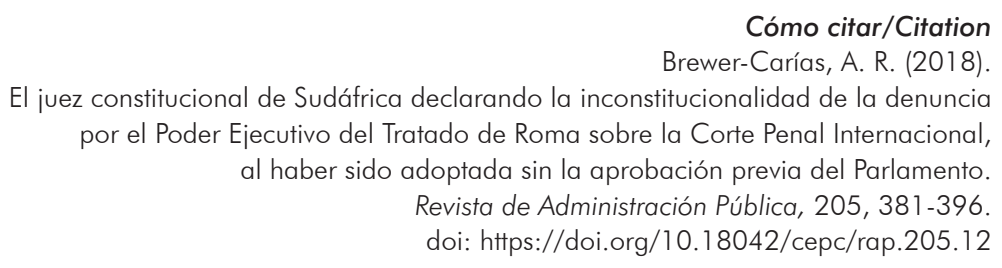

Resumen

La presente crónica se refiere a la sentencia de la High Court de Sudáfrica del 22 de febrero de 2017, que resolvió la demanda intentada por el partido Democratic Alliance contra el presidente de la República de Sudáfrica y sus ministros de Relaciones Internacionales y de Justicia, en la cual el Alto Tribunal declaró inconstitucional la denuncia ejecutiva del Tratado de Roma de la Corte Penal Internacional, porque había sido adoptada sin la previa aprobación del Parlamento, ordenando en consecuencia su revocatoria.

\section{Palabras clave}

Control de constitucionalidad; actos ejecutivos; tratados internacionales; denuncia; Parlamento; poderes de control. 


\section{Abstract}

This chronicle is referred to the decision of the High Court of South Africa, dated 22 February 2017, deciding the case Democratic Alliance against the President of the Republic of 'South Africa and its Ministers of International Relations and Justice and Correctional Services, in which it declared the unconstitutionality of the Executive decision to denounce the Rome Statute of the International Criminal Court, because issued without parliament approval, ordering its revocation.

\section{Keywords}

Judicial review; executive acts; international treaties; denounce; Parliament; control powers. 


\section{SUMARIO}

I. ANTECEDENTES: LA NEGATIVA DEL GOBIERNO DE SUDÁFRICA DE DETENER AL PRESIDENTE DE SUDÁN PERSEGUIDO POR LA CORTE PENAL INTERNACIONAL. II. BASES CONSTITUCIONALES DEL RÉGIMEN DE FORMACIÓN DE LOS TRATADOS. III. EL DEBATE SOBRE LAS BASES CONSTITUCIONALES DEL RÉGIMEN DE LA DENUNCIA DE LOS TRATADOS. IV. LA CONCLUSIÓN DE LA ALTA CORTE SOBRE LA NECESARIA APROBACIÓN PARLAMENTARIA PREVIA A LA DENUNCIA DE TRATADOS.

La Alta Corte (High Court) de Sudáfrica (Gauteng Division), con sede en Pretoria, actuando como juez constitucional, mediante sentencia de 22 de febrero de 2017 dictada en el caso: Democratic Alliance vs. Minister of International Relations, Minister of Justice and Correctional Services, the President of the Republic of 'South Africa (No: 83145/201)', declaró inconstitucional e inválida la decisión adoptada por el ministro de Relaciones Internacionales en fecha 19 de octubre de 2016 de denunciar el Tratado de la Corte Penal Internacional, y la decisión del gabinete ejecutivo de notificar dicha denuncia a la Secretaría General de Naciones Unidas, porque había sido adoptada sin la previa aprobación del Parlamento, ordenando en consecuencia a los demandados a revocar la denuncia del Tratado.

El Tratado que creó la Corte Penal Internacional fue adoptado en la Conferencia de Roma el 17 de julio de 1998, y fue ratificado por Sudáfrica el 27 de noviembre de 2000, habiendo sido aprobada dos años después, el 16 de agosto de 2002, mediante ley del Parlamento. Como se reafirmó en el preámbulo de la ley, la República de Sudáfrica asumió la obligación de llevar ante la justicia, sea ante las cortes nacionales cuando ello fuera posible, o ante la Corte Penal Internacional, a las personas que hayan cometido crímenes de guerra, contra la humanidad o de genocidio, incluyéndose en dicha ley los crímenes de apartheid; estableciendo además dicha ley los mecanismos legales

1 Véase el texto en https://www.dailymaverick.co.za/documents/document/DA-vMINISTER-OF-INTERNATIONAL-RELATIONS-20170219-FINAL.pdf. 
internos necesarios para asegurar la cooperación de Sudáfrica con dicha Corte Internacional.

\section{ANTECEDENTES: LA NEGATIVA DEL GOBIERNO DE SUDÁFRICA DE DETENER AL PRESIDENTE DE SUDÁN PERSEGUIDO POR LA CORTE PENAL INTERNACIONAL}

La decisión de denunciar el Tratado tuvo su origen remoto en la situación planteada en Sudáfrica en junio de 2015, con motivo de la negativa del gobierno de Sudáfrica de detener y entregar ante la Corte Penal Internacional, al presidente de Sudán, señor Omar Hassan Ahmad al- Bashir, quien asistía en Sudáfrica a la Cumbre de la Unión Africana, a pesar de que el mismo tenía en su contra dos órdenes de detención emitidas por la Corte Penal Internacional para su enjuiciamiento por crímenes de guerra, contra la humanidad y genocidio, todos relacionados con eventos ocurridos en la región de Darfur, en Sudán.

En esos días, en efecto, entre el 7 y el 15 de junio de 2015, en Sudáfrica se celebró la Cumbre de la Unión Africana (30th Ordinary Session of the Permanent Representatives Committee, the 27th Ordinary Session of the Executive Council and the 25th Ordinary Session of the Assembly of the African Union) con asistencia de los jefes de Estado de los países miembros, entre ellos del presidente de Sudán; hecho que originó para el gobierno la necesidad de resolver entre lo que consideró eran obligaciones internacionales solapadas, todas en el marco de las relaciones diplomáticas del momento.

Esas obligaciones eran, según se reseña en la sentencia, por una parte, la obligación de detener al presidente al-Bashir conforme a las previsiones del Tratado de Roma, y por la otra, la obligación que tenía el Estado ante la Unión Africana de asegurar la inmunidad a los participantes en la cumbre, tanto conforme al Acuerdo de sede de las mismas, como con las previsiones de la Convención General de Privilegios e Inmunidades de la Organización de la Unidad Africana de 1965, y las normas generalmente aplicables del derecho internacional, que reconocen la inmunidad de los jefes de Estado en ejercicio. El conflicto entre dichas obligaciones, además, se le presentaba al Gobierno en una circunstancia en la cual sus funcionarios estaban activamente involucrados en promover la paz, la estabilidad y el diálogo en esos países.

La decisión del gobierno de negarse a detener al presidente al-Bashir se fundamentó en el hecho de que este gozaba de inmunidad conforme al derecho internacional, habiendo sido ello lo que motivo una solicitud formulada por el South African Litigation Centre (SALC) ante la propia High Court para que dictase una orden declarando que la abstención del gobierno violaba 
la Constitución, solicitando, en consecuencia, que se ordenarse al Ejecutivo proceder a detener a al-Bashir y a entregarlo a la Corte Penal Internacional.

En ese caso, en 2015, la Alta Corte declaró efectivamente que la negativa del Gobierno había sido ilegal y contraria a la Constitución ${ }^{2}$, en una decisión que fue apelada por el Gobierno ante la Corte Suprema de Apelaciones. Esta Corte, en definitiva, declaró sin lugar dicha apelación ${ }^{3}$, habiendo sido dicha decisión recurrida por el Gobierno ante la Corte Constitucional, buscando dejarla sin efectos ${ }^{4}$. La Corte Constitucional, sin embargo, nada pudo decidir en el caso, pues el Gobierno retiró su petición antes de que tuviera lugar la audiencia final que había sido fijada para el día 22 de noviembre de 2016.

Un mes antes, en efecto, y a los efectos de no tener que cumplir en el futuro con las previsiones del Tratado de Roma, el mismo fue denunciado por el Gobierno el 19 de octubre de 2016, mediante notificación del Ministerio de Relaciones Internacionales al secretario general de Naciones Unidas, dándose así inicio al procedimiento de salida de Sudáfrica del mismo, la cual, conforme al art. 127.1 del mismo, debía tener efectos a los 12 meses siguientes de dicha notificación, es decir, en octubre de 2017.

Fue precisamente contra la decisión del gobierno de denunciar el Tratado de Roma cuando unos días después, el 24 de octubre de 2016, el partido político Democratic Alliance, que es el principal partido político de oposición en Sudáfrica, intentó una demanda contra el ministro de Relaciones Internacionales y Cooperación, responsable de firmar la notificación de denuncia del Tratado, contra el ministro de Justicia y Sercicios Correccionales, encargado de la aplicación del Tratado de Roma en el ámbito interno, y contra el presidente de la República de Sudáfrica, quien como jefe del Ejecutivo nacional, en los términos del art. 231.1 de la Constitución, es el responsable de la negociación y firma de los acuerdos internacionales.

La demanda fue intentada inicialmente como una acción directa de inconstitucionalidad por el partido Democratic Alliance ante la Corte Constitucional, y subsidiariamente, ante la High Court de Pretoria ${ }^{5}$. La demanda fue

2 Cita de la Corte: Southern Africa Litigation Centre v Minister of Justice and Constitutional Development \& others [2015] 3 All SA 505 (GP); 2015 (9) BCLR 1108 (GP); 2015 (2) SA 1 (GP).

3 Cita de la Corte: Minister of Justice and Constitutional Development \& others $v$ The Southern Africa Litigation Centre [2016] 2 All SA 365 (SCA); 2016 (4) BCLR 487 (SCA); 2016 (3) SA 317 (SCA).

4 Cita de la Corte: Minister of Justice and Constitutional Development and Others $v$ Southern Africa Litigation Centre and Others (CCT 75/16).

5 La demanda intentada por el partido Democratic Alliance fue secundada por diversas organizaciones no gubernamentales, en particular, por el Council for the Advance- 
rechazada por la Corte Constitucional por considerar que no era de interés de la justicia resolver la materia, quedando entonces el asunto bajo la competencia de la Alta Corte, la cual sí entró a considerar y decidir la materia mediante la sentencia de 22 de febrero de 2017, resolviendo, en definitiva, que la decisión del Ejecutivo nacional de denunciar el Tratado de Roma había sido inconstitucional e inválida, considerando que para poder haber sido adoptada, la misma debió haber sido previamente aprobada por el Parlamento.

Como el plazo de 12 meses para que la denuncia del Tratado se hiciese efectiva estaba transcurriendo, la Alta Corte aceptó la petición de urgencia para decidir sobre la inconstitucionalidad que se le había formulado, tomando en cuenta, además, el hecho de que los representantes del Gobierno demandados no habían presionado seriamente sobre la determinación o no de dicha la urgencia. Por ello, al decidir, la Corte consideró que «excepto si el asunto se decide ahora y la petición tiene mérito, el recurrente no va a obtener un efectivo remedio en ningún momento posterior».

\section{BASES CONSTITUCIONALES DEL RÉGIMEN DE FORMACIÓN DE LOS TRATADOS}

Las razones de fondo por las cuales el Gobierno procedió a tomar su decisión de denunciar el Tratado, para retirar a Sudáfrica de la jurisdicción de la Corte Penal Internacional, por supuesto, en ningún caso fueron consideradas por la Corte, limitándose su decisión a resolver la cuestión constitucional en el marco del régimen de los tratados internacionales conforme al principio de «la separación de poderes entre el Ejecutivo nacional y el Parlamento en materia de relaciones internacionales y conclusión de tratados», que se establece en el art. 231 de la Constitución de Sudáfrica de 1996.

En dicha norma se establece la competencia del Ejecutivo nacional para negociar y firmar acuerdos internacionales, que deben ser aprobados mediante ley para ser incorporados en el derecho interno, y la cuestión planteada constitucionalmente fue la de determinar si dicha competencia también incluía la de denunciar los acuerdos internacionales sin aprobación parlamentaria; y, además, determinar si era constitucionalmente permisible que el Ejecutivo nacional pudiera notificar la denuncia de un tratado, sin que antes se derogase la ley nacional que le dio efectos en el ámbito interno. En otros términos, la cuestión debatida ante la Corte fue la de determinar si el Ejecutivo nacional

ment of the South African Constitution (CASAC), el South African Litigation Centre (SALC); el Centre for Human Rights (CHR) y la Helen Suzman Foundation (HSF). 
tenía competencia para decidir denunciar el Tratado y ejecutar su decisión sin la intervención de la legislatura, y posteriormente pretender buscar una aprobación legislativa, y si podía ejecutar su decisión sin que se produjese previamente la derogación de la Ley de Aprobación del Tratado.

Para responder a estas cuestiones, la Corte partió de lo establecido en el mencionado art. 231 de la Constitución, donde se regula la forma como se concluyen los acuerdos internacionales, se hacen obligatorios en Sudáfrica y se incorporan al derecho interno, en particular, en las previsiones siguientes:

1. Es responsabilidad del Ejecutivo nacional la negociación y firma de todos los acuerdos internacionales.

2. Un acuerdo internacional es obligatorio en la república después de que ha sido aprobada por resolución de la Asamblea Nacional y del Consejo Nacional de Provincias, excepto que sea un acuerdo de los referidos en la subsección 3.

3. Un acuerdo internacional de naturaleza técnica, administrativa o ejecutiva, o un acuerdo que no requiere de ratificación ni de accesión, suscrito por el Ejecutivo nacional, es obligatorio para la República sin la aprobación de la Asamblea nacional y del Consejo Nacional de provincias, pero debe ser presentado en la Asamblea y el Consejo en un tiempo razonable.

4. Cualquier acuerdo internacional se convierte en ley en la República cuando se ha sancionado como ley por la legislacion nacional; pero las previsiones autoejecutivas de un acuerdo que ha sido aprobado por el Parlamento es ley en la República, salvo que sea inconsistente con la Constitución o una ley del parlamento.

Citando lo expresado por el juez Ngcobo CJ, en el caso Glenister ${ }^{6}$, la Alta Corte consideró que el esquema del art. 231 podía resumirse, partiendo del supuesto de que dicha norma «está enraizada en la doctrina de la separación de poderes, en particular, los controles y equilibrios entre el ejecutivo y el legislativo", contemplando tres pasos claramente establecidos que deben darse en relación con los acuerdos internacionales, con consecuencias legales diferentes:

Primero, asigna al Ejecutivo nacional la autoridad para negociar y firmar los acuerdos internacionales. Pero un acuerdo internacional firmado por el Ejecutivo no obliga automáticamente a la República excepto si el acuerdo es de naturaleza técnica, administrativa o ejecutiva. Para producir ese resultado requiere, segundo, la aprobación por resolución del Parlamento. [...] y tercero, un acuerdo internacional se convierte en ley en la República cuando se sanciona como ley por la legislación nacional.

6 Cita de la Corte: Glenister $v$ President of the Republic of South Africa and others 2011 (3) SA 347; 2011 (7) BCLR 651 (CC) (Glenister II). 
De ello, consideró la Alta Corte que conforme a dicho art. 231 constitucional no hay duda de que el poder para conducir las relaciones internacionales y suscribir tratados fue conferida constitucionalmente al Ejecutivo nacional (art. 231.1); pero que dicho poder está limitado por los arts. 231.2 y 231.4, que exigen al Ejecutivo comprometer al Parlamento en el proceso, delineando claramente los poderes entre el Ejecutivo nacional y el Parlamento. El único caso en el cual el Ejecutivo nacional tiene poder para obligar al país en acuerdos internacionales sin intervención parlamentaria es el regulado en el art. 231.3, que no era el caso relativo a la decisión. Respecto de cualquier otro acuerdo internacional, para que pueda ser obligatorio en el país debe ser aprobado por el Parlamento en los términos del art. 231.2. En consecuencia, concluyó la Corte, una vez que el Parlamento aprueba un acuerdo internacional, el país queda comprometido internacionalmente con el mismo. En el ámbito interno, el proceso se completa cuando el Parlamento sanciona el acuerdo internacional como ley nacional en los términos del art. 231.4 de la misma Constitución.

\section{EL DEBATE SOBRE LAS BASES CONSTITUCIONALES DEL RÉGIMEN DE LA DENUNCIA DE LOS TRATADOS}

Una vez hecho el resumen anterior sobre lo indicado en el art. 231 de la Constitución en materia de conclusión de tratados, la Alta Corte reconoció que sobre el mismo, si bien por lo que se refería a dicho proceso en el caso no había debate alguno, en cambio sí lo había por lo que se refería al proceso de denuncia de los tratados, cuando el país decide retirarse de un acuerdo internacional.

Sobre esto, el partido demandante argumentó en el proceso que, de acuerdo con los términos del art. 231.2, es el Parlamento el que debe aprobar un acuerdo internacional antes de que pueda ser obligatorio en Sudáfrica, la consecuencia de ello es que también debe ser el Parlamento el que debe decidir si un acuerdo internacional debe cesar de ser obligatorio en el país, antes de que el Ejecutivo pueda enviar la notificación de su denuncia. Por su parte, los representantes del Gobierno argumentaron lo contrario en el sentido de considerar que la previa aprobación parlamentaria no es requerida para notificar la denuncia del tratado, ya que el art. 231 no contiene previsión alguna al respecto. Es decir, básicamente, el argumento de los representantes del Gobierno fue que como no había previsión expresa en el art. 231 en tal sentido, tal exigencia era injustificada.

El argumento de los representantes del Gobierno, como lo resumió la sentencia, se basó en las cuatro premisas siguientes: 
Primero, se argumentó que en virtud de que el Ejecutivo nacional, y no el Parlamento, es el que tiene el rol primario en la conducción de las relaciones internacionales para concluir tratados, el requerimiento legal de la aprobación previa del Parlamento, no estando explícito en la Constitución, no puede considerarse implícito en ella. Por tanto, como se ha interpretado por la Corte Constitucional, considerar al Parlamento como el órgano primario decisor en materia de tratados sería contrario al art. 231 de la Constitución.

Segundo, relacionado con lo anterior, también se argumentó que como la competencia original para concluir tratados no es del Parlamento sino del Ejecutivo nacional, la aprobación parlamentaria solo es requerida a los solos efectos de hacer que el tratado concluido tenga efectos obligatorios, quedando la conclusión de tratados como la función primaria del Ejecutivo nacional. De ello, siguió el argumento de los representantes del Gobierno, que, como la forma de expresión de la aprobación de un tratado es a través de la ratificación, en este contexto significa la confirmación formal del consentimiento expresado por el Ejecutivo nacional, en consecuencia, la denuncia del tratado corresponde también al Ejecutivo, no siendo necesaria para ello la aprobación parlamentaria, la cual solo se exige para asegurar los efectos obligatorios del tratado una vez firmado. En definitiva, si la conclusión de un tratado corresponde básicamente al Ejecutivo nacional, igualmente, el deshacer el tratado también debe ser una competencia constitucional del Ejecutivo. Adicionalmente se argumentó que como los tratados no son negociados ni firmados por el Parlamento sino por el Ejecutivo, el Parlamento no puede decidir denunciar un tratado, no siendo por ello requerida la aprobación parlamentaria para la denuncia del tratado.

Tercero, igualmente se argumentó que en el campo del derecho internacional para la notificación de la denuncia de un acuerdo internacional no se requiere de aprobación parlamentaria, citándose al efecto el art. 56 de la Convención de Viena sobre derecho de los Tratados de 1969, en la cual se basa el art. 127 del Tratado de Roma, el cual solo y únicamente dispone sobre la notificación de la denuncia por parte del jefe de Estado, o de Gobierno o del ministro de Relaciones Exteriores, o de un representante del Estado, sin que se requiera aprobación, ratificación o confirmación parlamentaria. Deducir lo contrario, se argumentó, sería inconsistente con el derecho internacional y con el requerimiento constitucional de interpretar la Constitución y la ley de Sudáfrica conforme al derecho internacional.

Cuarto, también se argumentó que la aprobación parlamentaria es solo requerida para los acuerdos internacionales, por lo que siendo la denuncia un acto unilateral, la misma no califica como acuerdo internacional para que pueda argumentarse que también requiere de aprobación parlamentaria.

En relación con estos argumentos de los representantes del Gobierno, la Alta Corte expresó que no tenía dificultad alguna en aceptar la proposición 
conforme a la cual y de acuerdo con el esquema constitucional del art. 231, es responsabilidad del Ejecutivo nacional tanto el desarrollo de políticas, teniendo la iniciativa legislativa para implementarlas, como la conducción de las relaciones internacionales, materia en relación con la cual las Cortes no tienen rol alguno.

Sin embargo, la Corte manifestó su desacuerdo con la orientación general de los argumentos formulada por los representantes del Gobierno, los cuales los sintetizó así:

En los términos de los artículos 231.1 y 231.2 de la Constitución el Ejecutivo nacional, primero negocia y firma el acuerdo internacional. Seguidamente el Parlamento aprueba el acuerdo para obligar al país. El proceso de denuncia debe seguir la misma ruta, primero con el Ejecutivo nacional tomando la decisión, seguida de la aprobación parlamentaria. Conforme a este argumento, la notificación de la denuncia es un acto ejecutivo que en términos del artículo 231.1, es equivalente o parecido a la conclusión y firma del tratado durante el proceso de negociación, que no requiere de previa aprobación parlamentaria, pero que puede ser subsecuentemente ratificado.

La Corte afirmó tajantemente no estar de acuerdo con lo anterior. Al contrario de ello, sostuvo en su decisión que:

Una notificación de denuncia, conforme a una apropiada interpretación del artículo 231.1, es equivalente a la ratificación, la cual requiere de previa aprobación parlamentaria en los términos del artículo 231.2. Como fue correctamente argumentado por el demandante, el acto de firmar un tratado y el acto de notificar su denuncia son diferentes en sus efectos. El primero no tiene consecuencias jurídicas directas, mientras que, en contraste, la entrega de la notificación de denuncia tiene un efecto concreto en derecho internacional, pues termina con las obligaciones del tratado. [...] Aun cuando [conforme al artículo 127.1 del Tratado de Roma] la denuncia no surte efectos sino luego de un año, la notificación constituye a nivel internacional una decisión obligatoria, incondicional y final de salida del Tratado de Roma.

Adicionalmente, la Alta Corte manifestó su desacuerdo con el argumento del representante del Gobierno de que la participación del Parlamento en el proceso de decidir lo concerniente a la denuncia del Tratado de Roma sería inconsistente con el derecho internacional, pues la comunicación de la denuncia debe estar firmada por un alto funcionario del Estado. El art. 127 del Tratado de Roma, que el representante del Gobierno sostuvo que estaba basado en el art. 56 de la Convención de Viena de 1969, simplemente requiere que la notificación de la denuncia esté firmada por el jefe de Estado, el jefe de gobierno o el ministro de Asuntos Exteriores u otro representante del Estado 
involucrado. Conforme a una interpretación apropiada, sostuvo la Corte, esto implica que la notificación debe ser firmada por un alto funcionario del Estado que sea debidamente autorizado para ello, de manera que el secretario general y la Corte Penal Internacional puedan considerar dicha notificación como auténtica. Pero este artículo «no tiene el propósito de imponer a los Estados miembros cómo y por quién debe ser tomada la decisión de denuncia». En resumen, la Corte sostuvo que:

[...] la previsión no tiene nada que ver respecto de quién tiene la competencia para adoptar la decisión de denuncia del tratado, sino que está destinada a indicar el funcionario gubernamental que firma y envía la notificación a las Naciones Unidas, luego de que la autoridad competente (sea el Ejecutivo nacional o la legislatura) ha tomado la decisión de denunciar. En consecuencia, el artículo no tiene relación con la toma de la decisión en sí misma. De allí que la necesidad de la participación del Parlamento en la consideración de la conclusión de los tratados y, por analogía, la cuestión de si la denuncia del Tratado de Roma sea parte del derecho interno y no puede interpretarse en forma inconsistente con el derecho internacional.

En definitiva, la Alta Corte consideró que:

[...] ciertamente es correcto que conforme al derecho internacional la notificación de denuncia de un acuerdo internacional no requiere de aprobación parlamentaria ${ }^{7}$, pero, sin embargo, la cuestión de determinar quién entre el Ejecutivo nacional y el Parlamento debe decidir sobre la denuncia, debe ser resuelta de acuerdo con el derecho interno. Es un asunto interno en el cual el derecho internacional nada ha regulado ni puede regular.

Por otra parte, y en cierta forma aplicando el principio general del paralelismo de las formas, aplicable a todas las actuaciones de los órganos del Estado, la Corte consideró que:

[...] debía tenerse en cuenta que si bien es necesaria la previa aprobación parlamentaria antes de que el instrumento de ratificación sea depositado en las Naciones Unidas, desde esa perspectiva, es difícil no aceptar que el procedimiento contrario de denuncia no deba ser objeto del mismo proceso parlamentario. La necesaria deducción, que deriva de la interpretación apropiada del artículo 231, es que el Parlamento retiene su poder de determinar si el Estado debe permanecer obligado por un tratado internacional. Esto es necesario para dar sentido a la clara separación de poderes entre el Ejecutivo nacional y la Legislatura inserta

7 Cita de la Corte: véase el art. 127 del Tratado de Roma, y su base el art. 56 de la Convención de Viena sobre derecho de los Tratados. 
en la norma. Si es el Parlamento el que determina si un acuerdo internacional obliga al país, entonces debe considerarse constitucionalmente insostenible pretender que el Ejecutivo nacional pueda terminar unilateralmente dicho acuerdo.

Lo anterior lo indicó la Alta Corte para concluir, citando lo expresado en el caso Glenister II (para 96) resuelto por la Corte Constitucional, que:

[...] la aprobación de un acuerdo internacional en los términos del artículo 231.2 crea un contrato social entre el pueblo de Sudáfrica, a través de sus representantes electos en la Legislatura, y el Ejecutivo nacional. Ese contrato social origina derechos y obligaciones expresadas en el acuerdo internacional, por lo que sería por tanto una evidente y manifiesta anomalía, que el Ejecutivo nacional pudiera terminar con esos derechos y obligaciones, sin obtener la aprobación del pueblo de Sudáfrica.

\section{LA CONCLUSIÓN DE LA ALTA CORTE SOBRE LA NECESARIA APROBACIÓN PARLAMENTARIA PREVIA A LA DENUNCIA DE TRATADOS}

Como consecuencia de lo anterior, y partiendo del principio jurisprudencial de que «cuando una previsión constitucional o legal confiere poderes para hacer algo, esa previsión necesariamente también confiere poder para deshacer ${ }^{8}$, la Alta Corte concluyó afirmando que en el contexto del caso, si el poder de obligar al país con el Tratado de Roma se le confiere al Parlamento, también le corresponde al Parlamento el poder de decidir si un tratado internacional debe cesar de obligar al país. Por ello, la Corte concluyó su decisión en la materia afirmando que conforme a la interpretación literal del art. 231.2 de la Constitución:

Sudáfrica puede retirarse del Tratado de Roma solo con la aprobación del parlamento y después de que se derogue la ley de aprobación [del Tratado].

La Corte consideró que esta interpretación del art. 231 de la Constitución es la más adecuada constitucionalmente, dando efectos a la separación de poderes tan claramente delineada en la norma, no siendo impedimento alguno para sostenerla el hecho de que la norma no establezca expresamente

8 Cita de la Alta Corte: Masetlha v President of the Republic of South Africa and Another 2008 (1) SA 566 (CC) para 68. 
que solo el Parlamento tiene el poder de decidir sobre el retiro del Tratado de Roma.

Es más, sobre esto último, el hecho de que la Constitución haya regulado el poder del Ejecutivo para negociar y concluir acuerdos internacionales, y nada haya establecido sobre el poder de terminar con dichos acuerdos, lo justificó la Corte en su sentencia, en el hecho de que la ausencia de tal regulación lo que confirma es el principio mismo de legalidad y de la necesidad de competencia legal expresa que rige la actuación de los órganos del Poder Ejecutivo para ello, la Corte dio la siguiente razón:

El Ejecutivo nacional, como instrumento ejecutivo del Estado, necesita tener competencia para actuar, debiendo fluir la misma de la Constitución o de una ley del Parlamento. El Ejecutivo nacional solo puede ejercer los poderes y cumplir las funciones que se le confieren expresamente en la Constitución o en las leyes que sean consistentes con la Constitución?. Este es el requerimiento básico del principio de legalidad y del Estado de derecho. Por ello, la ausencia de una previsión en la Constitución o en cualquier otra ley que confiera poder al Ejecutivo para terminar acuerdos internacionales, lo que confirma es que tal poder no existe excepto y hasta cuando el Parlamento legisle en tal sentido. Por tanto, en esta materia no hay ninguna lacuna u omisión.

Por otra parte, en cuanto al fondo en esta materia, la propia Corte consideró que hubiese sido:

[...] imprudente, si la Constitución hubiese dado tal poder al Ejecutivo de denunciar los acuerdos internacionales y en consecuencia terminar con los derechos y obligaciones existentes, sin obtener previamente la aprobación por parte del Parlamento. Eso hubiese significado conferir poderes legislativos al Ejecutivo, en una clara ruptura del principio de la separación de poderes y del Estado de derecho. Sobre esta base, el Ejecutivo nacional no tiene y nunca fue la intención de que tuviera el poder de terminar con los acuerdos internacionales existentes, sin la previa aprobación parlamentaria.

Por todo ello, en otra parte de la sentencia, la Corte consideró en general que el ejercicio del poder público, incluyendo la conducción de las relaciones internacionales, debe siempre realizarse de acuerdo con la Constitución ${ }^{10}$, y que

$9 \quad$ Cita de la Alta Corte Fedsure Life Assurance Ltd v Greater Johannesburg Transitional Metropolitan Council 1999 (1) SA 374 (CC) at paras 56-56; President of RSA v SARFU 2000(1) SA 1 (CC) at para 148; Mansigh v General Council of the Bar and Others 2014 (2) SA 26 (CC) at para 25.

10 Cita de la Corte: Pharmaceutical Manufacturers Association of SA and Another: In re Ex parte President of the Republic of South Africa and Others 2000 (2) SA 674 (CC) para 20. 
si bien Sudáfrica, conforme al art. 231 de la Constitución, había ratificado el Tratado de Roma y había sancionado la Ley aprobatoria del mismo, en cuanto a la notificación de la denuncia del Tratado, si bien había sido firmada y notificada como acto ejecutivo en el marco de las relaciones internacionales, como manifestación del ejercicio del poder público debía cumplir con el principio de legalidad y esta sujeción al control constitucional ${ }^{11}$.

De todo lo anterior, la Alta Corte concluyó afirmando que como en la estructura del art. 231, el Ejecutivo nacional requiere de la previa aprobación parlamentaria para obligar a Sudáfrica en un acuerdo internacional, no hay razón alguna para que la salida de tal acuerdo sea diferente. Por ello, en el caso, el Ejecutivo nacional no tenía poder para enviar la notificación de denuncia sin obtener la previa aprobación parlamentaria, siendo la inevitable conclusión que la notificación de la denuncia del Tratado de Roma «requería del imprimatur del Parlamento» antes de ser consignada ante las Naciones Unidas. En consecuencia, la decisión del Ejecutivo nacional de enviar la notificación de la denuncia del Tratado sin obtener la previa aprobación parlamentaria violó el art. 231.2 de la Constitución, y la doctrina de la separación de poderes inserta en tal norma.

Con base en esta decisión, la Corte concluyó rechazando la posibilidad de que la aprobación parlamentaria exigida en la Constitución pudiera darse ex post facto, como lo pretendieron los representantes del Gobierno al haberse en efecto sometido al Parlamento la consideración de la denuncia del Tratado de Roma y un proyecto de ley derogatoria de la ley aprobatoria, para evitar la declaratoria de inconstitucionalidad e invalidez de la denuncia del Tratado de Roma.

La Corte, en efecto, consideró que estando en la materia implicado el principio de la separación de poderes, en virtud de que el Ejecutivo nacional ejerció un poder que no tiene asignado constitucionalmente, su conducta fue inválida y no tiene efecto en derecho ${ }^{12}$, por lo que cualquiera sea lo que el Parlamento decida respecto de la subsecuente solicitud hecha por el Ejecutivo nacional para obtener su aprobación de la notificación de denuncia del Tratado, no puede remediar la invalidez.

11 Cita de la Corte: Kaunda and others v President of the Republic of South Africa 2005 (4) SA 235 (CC) paras 78- 80,178,191 and 228; Minister of Defence and Military Veterans $v$ Motau and others 2014 (5) SA 69 (CC) para 69; National Treasury $v$ Opposition to Urban Tolling Alliance 2012 (6) SA 223 (CC) para 64.

12 Cita de la Corte: Kruger v President of the Republic of South Africa and others 2009 (1) SA 417 (CC) para 52. 
Citando a Hoexter ${ }^{13}$, la Corte afirmó que «[un] acto inválido, siendo nulo, no puede ser ratificado, convalidado o corregido", estimando incluso que tal principio permanece vigente aun cuando, como fue el caso de la denuncia del Tratado de Roma, los efectos de la misma no se produjeron de inmediato y solo se producirían en octubre de 2017, pues estaba en curso el lapso de 12 meses previsto en el Tratado. Respecto de ello, la Corte concluyó indicando que el Parlamento no tiene poder alguno para remediar la invalidez que ya afectó a la notificación de la denuncia, lo cual, sin embargo, no afecta la validez de decisión del ministro de Justicia de haber presentado ante el Parlamento el proyecto de ley de derogación de la ley de aprobación del Tratado.

Por todo lo anterior, como antes se indicó, la Alta Corte de Sudáfrica, con sede en Pretoria, declaró que la decisión del Ejecutivo nacional de consignar la notificación de denuncia del Tratado de Roma para provocar la salida de Sudáfrica del mismo, adoptada sin la previa aprobación parlamentaria, es inconstitucional e inválida, dándole a la decisión efectos retroactivos, es decir, al momento en el cual se entregó la notificación, ordenando en consecuencia al Ejecutivo nacional a revocarla.

Por último, debe mencionarse que al decidir el caso, la Corte precisó que conforme a su decisión, "lo que había sido inconstitucional e inválido fue la implementación de la decisión de enviar la notificación de dicha denuncia, sin la previa aprobación parlamentaria», absteniéndose de "expresar su opinión sobre la decisión política sustantiva del Ejecutivo nacional de denunciar el Tratado de Roma», considerando que "no sería apropiado el declarar esa decisión inconstitucional como decisión aislada», pues «no hay nada patentemente inconstitucional, al menos en esta etapa, sobre la decisión política del retiro del Estado del Tratado de Roma, ya que adoptar esa decisión está entre sus poderes y competencias.

Por ello, la Corte consideró que a los efectos de su decisión «era suficiente y efectivo, como medida justa y equitativa, la declaración de invalidez de la notificación de la denuncia del Tratado, acompañada de la orden de retirar dicha notificación", decisión que adoptó la Ata Corte conforme a lo dispuesto en el art. 172.1 de la Constitución que establece que cuando una corte de justicia, conforme a sus poderes en materia constitucional, debe declarar que una ley o conducta que es inválida por ser inconsistente con la Constitución, puede proceder a acordar "cualquier orden justa y equitativa», conforme al

13 Cita de la Corte: Administrative Law in South Africa $2^{\text {nd }}$ ed (2012) at 547. See also S v Cebekulu 1963 (1) SA 482 (T) at 483; Montshioa and Another v Motshegare 2001 (8) BCLR 833 (B) para 24. 
art. 172.1.b de la Constitución, como fue la de ordenar al Ejecutivo nacional a revocar la denuncia del Tratado de Roma.

Luego de la sentencia, el gobierno, en cumplimiento de lo resuelto por el juez constitucional, notificó formalmente a la Secretaría General de las Naciones Unidas que «con el fin de cumplir la sentencia, queda revocada la petición de salida del Estatuto de Roma de la Corte Penal Internacional con efecto inmediato» ${ }^{14}$.

14 Véase la reseña: «Sudáfrica retira su petición de abandonar la Corte Penal Internacional,» Agencia EFE Johannesburgo 8 de marzo de 2017, en https://www.efe.com/efe/ america/portada/sudafrica-retira-su-peticion-de-abandonar-la-corte-penal-internacional/20000064-3201050. 